LA-UR-96- -54

Title:

Macroscopic Time and Altitude Distribution of Plasma Turbulence Induced in Ionospheric Modification Experiments

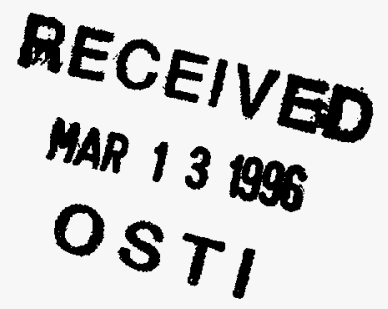

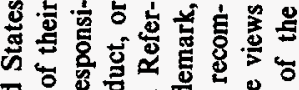

马

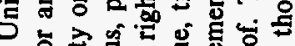

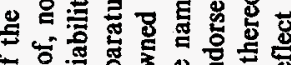

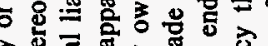

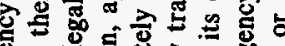

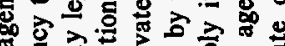

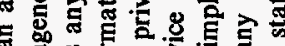

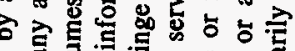

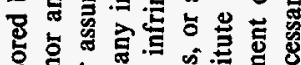

与

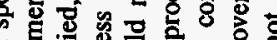

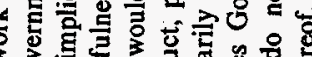

है동

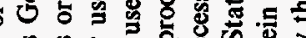

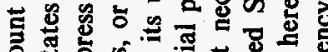

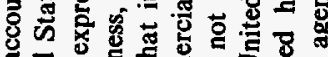

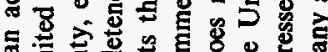

Submitted to:

Author(s):

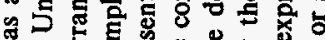

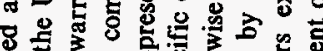

语

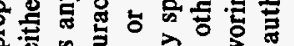

空

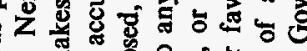

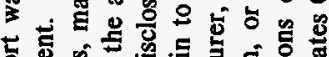

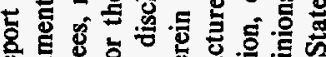

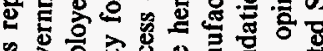

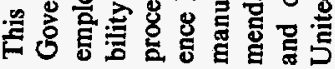

Harvey Rose, T-13

Donald Dubois, T-13

David Russell, Lodestar Research

Corporation, Boulder, CO

Alfred Hanssen, University of Tomosø, Norway

DOE Office of Scientific and Technical Information

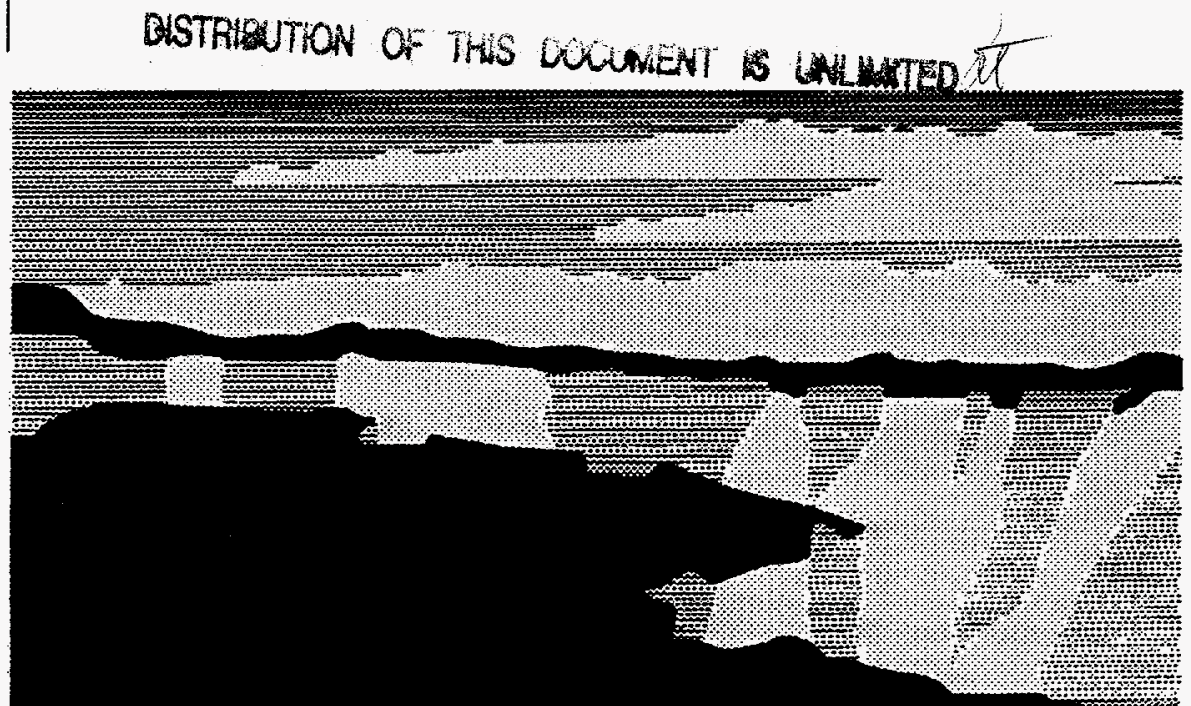

Los Alamos National Laboratory, an affirmative action/equal opportunity employer, is operated by the University of Calliomia for the U.S. Department of Energy under contract W-7405-ENG-36. By acceptance of this article, the publisher recognizes that the U.S. Govemment retains a nonexclustie, royaltyfree license to publish or reproduce the published form of this contribution, or to allow others to do so, for U.S. Government purposes. The Los Alamos National Laboratory requests that the publisher identily this article as work pertormed under the auspices of the U.S. Department of Energy. 
DISCLAIMER

Portions of this document may be illegible in electronic image products. Images are produced from the best available original document. 


\title{
Macroscopic Time and Altitude Distribution of Plasma Turbulence Induced in Ionospheric Modification Experiments
}

Harvey Rose*, Donald Dubois, David Russell (Lodestar Research Corporation), and Alfred Hanssen (University of Troms $\emptyset$, Norway)

\begin{abstract}
This is the final report of a three-year, Laboratory-Directed Research and Development (LDRD) project at the Los Alamos National Laboratory (LANL). This research concentrated on the time dependence of the heater, inducedturbulence, and electron-density profiles excited in the ionosphere by a powerful radio-frequency heater wave. The macroscopic density is driven by the ponderomotive pressure and the density self-consistently determines the heater propagation. For typical parameters of the current Arecibo heater, a dramatic quasi-periodic behavior was found. For about $50 \mathrm{~ms}$ after turn-on of the heater wave, the turbulence is concentrated at the first standing-wave maximum of the heater near reflection altitude. From 50-100 ms the standingwave pattern drops by about $1-2 \mathrm{~km}$ in altitude and the quasi-periodicity reappears at the higher altitudes with a period of roughly $50 \mathrm{~ms}$. This behavior is due to the half-wavelength density depletion grating that is set up by the ponderomotive pressure at the maxima of the heater standing-wave pattern. Once the grating is established the heater can no longer propagate to higher altitudes. The grating is then unsupported by the heater at these altitudes and decays, allowing the heater to propagate again and initiate another cycle. For stronger heater powers, corresponding to the Arecibo upgrade and the HAARP heater now under construction, the effects are much more dramatic.
\end{abstract}

\section{Background and Research Objectives}

The objective of this research is to develop theoretical predictions for the macroscopic spatial and temporal distribution of turbulence induced in the ionosphere by powerful highfrequency (hf) radio waves. In the past few years there has been a dramatic improvement in the quality of experiments measuring the plasma turbulence induced near the reflection layer

*Principal investigator, e-mail: har@lanl.gov 
of the ionosphere by such waves [1-4]. These experiments, with unprecedented space and time resolution, have been strongly influenced by a new theoretical description of this turbulence developed at Los Alamos [5-7]. Ground-based experiments have been carried out at the radar and heater (hf) facilities at Arecibo, Puerto Rico, and at Troms $\emptyset$, Norway.

The induced "Langmuir turbulence" is a chaotic, complex system characterized by highfrequency turbulent fluctuations near the electron plasma frequency and low-frequency fluctuations in the range of ion acoustic frequencies. Our research has shown that under a wide range of conditions a significant part of the turbulent energy is carried by "caviton" excitations consisting of a trapped high-frequency electric field in a self-consistent density cavity. These cavitons undergo a life history of nucleation [8,9], collapse [10-12] and burnout [13]. In the last stage the trapped electric field energy is given up to the acceleration of superthermal electrons. The caviton excitations interact with free Langmuir waves and ion acoustic waves which also carry part of the turbulent energy [5].

In the first tens of ms after the turn-on of the hf heater, the observed turbulent spectra are in excellent agreement-with respect to spectral shape and altitude distribution-with theoretical predictions $[\mathbf{1 , 4 , 5 , 7 ]}$. This was the first dramatic prediction of our Strong Langmuir Turbulence (SLT) theory, which is totally different than previous Weak Turbulence Approximation (WTA) predictions. For this early-time turbulence, localized in the first few heater interference maxima below the reflection layer, the WTA fails completely. The SLT theory also predicts that at lower altitudes (about 1-2 km below reflection for typical Arecibo conditions), a rather discrete decay plus cascade spectrum should be observed in the undisturbed electron density profile $[6,14]$. This spectrum is qualitatively similar to WTA predictions but quantitatively different in the SLT theory.

For many years it has been known that this sharp decay-cascade spectrum develops at later heating times. (The time delay decreases with increasing heater power being about $30 \mathrm{~ms}$ at full Arecibo power.) The surprise from recent altitude-resolved observations $[2,3,4]$ is that this decay-cascade turbulence does not occur at the altitudes predicted assuming the background ionospheric density profile remains undisturbed.

\section{Importance to LANL's Science and Technology Base and National R\&D Needs}

The hf modification of the ionosphere is an international program. The heater power has recently been upgraded at Troms $\emptyset$ and at Arecibo which will make new parameter regimes accessible. A new hf heating facility and diagnostic radars are in the early construction step in Alaska as part of the HAARP (Highpower Active Auroral Research Project). We collaborate 
with a group at the University of California, Los Angeles, that operates the HIPAS facility in Alaska and also performs experiments at Arecibo. The hf heating experiments provide a unique outdoor laboratory that has a well-characterized plasma, with long space and time scales, and that is unaffected by boundaries. The quality of the diagnostics allows detailed comparison of theory and experiment. To understand these phenomena in the ionosphere and to make a valid comparison of theory and experiment, the unique and variable properties of the ionospheric plasma and the special observational diagnostics must be understood.

The SLT theory has universal scaling properties that makes it applicable to the very different space-time scales relevant to laser-plasma interactions. There are direct lessons to be learned for problems of interest to the Department of Energy (DOE) inertial confinement fusion (ICF) program. The hf-driven ionosphere is, in fact, a rather high-energy-density plasma in the sense that the electromagnetic energy density can be a large fraction of the thermal energy density. Of particular common interest to ICF are the superthermal electrons generated by caviton collapse [15] in SLT and observed in the ionosphere by airglow measurements and enhanced natural plasma lines.

This research makes extensive use of Laboratory supercomputing capabilities. In collaboration with other Laboratory researchers and researchers at the University of lowa, we have recently developed particle-in-cell and Vlasov codes for computing the properties of superthermal electrons. At a fundamental level the study of SLT involves the gamut of phenomena of interest to modern nonlinear complex systems studies. These include local coherent (soliton-like) excitations, chaos, and self-organization. Los Alamos has an ongoing experimental program that uses the facilities at Arecibo, HIPAS, and Troms $\emptyset$. We have collaborated with them (as well as with researchers at the Naval Research Laboratory, Cornell and Dartmouth) on the design and interpretation of the National Aeronautics and Space Administration (NASA) sounding-rocket and satellite probes.

\section{Scientific Approach and Results}

This research is simplified by the fact that the space and time scales of the local Langmuir turbulence are much smaller than the scales governing the macroscopic space-time evolution of the self-consistent heater field. The heater field is governed mainly by the variation of the electron density profile on spatial scales of the order of a wavelength, which near reflection altitude is greater than $100 \mathrm{~m}$. The correlation lengths of the Langmuir turbulence fluctuations are estimated to be about $1 \mathrm{~m}$ or less.

If the local heater field $\mathrm{E}_{\mathrm{H}}(\mathrm{z}, \mathrm{t})$ and mean electron density $<\mathrm{n}(\mathrm{z}, \mathrm{t})>$ are known at a given altitude $\mathrm{z}$ and time $\mathrm{t}$, the local turbulence properties can be calculated. (The mean density 
$<\mathrm{n}(\mathrm{z}, \mathrm{t})\rangle$ is averaged over spatial scales large compared to turbulence correlation lengths but small compared to a heater wavelength.) The local calculations can be done with an array of spectral simulation codes in one or two dimensions, which we have developed in past research. By the end of this three-year project, a three-dimensional spectral code will be available that exploits the highly parallel CM-5 computing resource in the Advanced Computing Laboratory at Los Alamos.

The self-consistency problem is that $\mathrm{E}_{\mathrm{H}}(\mathrm{z}, \mathrm{t})$ and $\langle\mathrm{n}(\mathrm{z}, \mathrm{t})>$ respond to the induced local turbulence. At heater turn-on, before the turbulence has time to fully develop, $\langle\mathrm{n}(\mathrm{z}, \mathrm{t})\rangle=$ $\mathrm{n}_{\mathrm{O}}(\mathrm{z})$, the background ionospheric density profile. [Using new techniques, Djuth et al. (private communication) have found that $\mathrm{n}_{\mathrm{O}}(\mathrm{z})$ at Arecibo is smooth on the level of $0.1 \%$ fluctuations over about $100 \mathrm{~m}$ in altitude.] The initial standing wave heater profile $\mathrm{E}_{\mathrm{H}^{\circ}} \mathrm{(z,t)}$ is easily calculated knowing $\mathrm{n}_{\mathrm{O}}(\mathrm{z})$ and is roughly an Airy function with maxima (near reflection) separated by about $150-200 \mathrm{~m}$. Recent measurements by Djuth et al. of the induced turbulence at later times following heater turn-on give dramatic proof that the turbulence occurs in layers corresponding to the heater maxima. These layers are observed to persist for tens of seconds. The ponderomotive pressure of the fluctuating Langmuir electric fields of the turbulence and the weaker pressure of the heater wave itself will tend to expel electrons and ions from the region of the heater maxima. Thus, $\left\langle\mathrm{n}(\mathrm{x}, \mathrm{t})>\right.$ will be reduced in these regions relative to $\mathrm{n}_{\mathrm{O}}(\mathrm{z})$ and the heater profile must readjust.

We developed a mesoscale code in collaboration with Alfred Hanssen that is designed to treat the self-consistent system of heater field, average density and local turbulence in an isothermal approximation. The code uses data from a large number of local turbulence simulations to calculate the response of $\langle\mathrm{n}(\mathrm{x}, \mathrm{t})\rangle$ to a continuous range of values of $\mathrm{E}_{\mathrm{H}}(\mathrm{z}, \mathrm{t})$. We used this mesoscale code to calculate the macroscopic self-consistent heater, density and turbulence profiles as a function of $\mathrm{z}$ and tincluding the long-time ( $\geq 1$ second) equilibrium profiles. We believe that the resulting modified density profile is the explanation of the several existing discrepancies between observations and the predictions of the turbulence theory in an unmodified profile.

A two-dimensional function, which is the local averaged Langmuir wave-energy density as a function of local heater strength $E_{H}$ and local self-consistent density, has been constructed from a large number of homogeneous turbulence simulations including those carried out by our collaborator, Alfred Hanssen. We now believe that the two-dimensional function has been sufficiently characterized. The function is solved in conjunction with the wave equation for the heater field to determine the self-consistent heater and electron density profile. The equations used are given in [16] where preliminary results are presented. 
During the final year of this project our research concentrated on the time dependence of the heater, induced-turbulence, and electron-density profiles excited in the ionosphere by a powerful hf heater wave. The mesocaling theory developed earlier in this project was used for these calculations. This procedure used a series of one-dimensional, homogeneous, turbulence simulations to obtain the induced turbulent ponderomotive pressure as a function of local heater intensity and local electron density. The macroscopic density is driven by this ponderomotive pressure and the density self consistently determines the heater propagation.

A dramatic quasi-periodic behavior was found for typical parameters of the current Arecibo heater. For about $50 \mathrm{~ms}$ after turn on of the heater wave, the turbulence (as observed) is concentrated at the first standing wave maximum of the heater near reflection altitude. From $50-100 \mathrm{~ms}$ the standing wave pattern drops by about $1-2 \mathrm{~km}$ in altitude and the quasiperiodicity reappears at the higher altitudes with a period of roughly $50 \mathrm{~ms}$. This behavior is due to the half-wavelength density depletion grating that is set up by the ponderomotive pressure at the maxima of the heater standing wave pattern. Once the grating is established the heater can no longer propagate to higher altitudes. The grating is then unsupported by the heater at these altitudes and decays allowing the heater to propagate again and initiate another cycle. For stronger heater powers, corresponding to the Arecibo upgrade and the HAARP heater now under construction, the effects are much more dramatic. The important lesson here is that the heater and ionospheric density profiles are not known a priori, as usually assumed, after the first cycle and must be self-consistently determined.

\section{References}

[1] P. Y. Cheung, A. Y. Wong, T. Tanikawa, J. Santoru, D. F. DuBois, H. A. Rose and D. Russell, "Short Time Scale Evidence for Strong Langmuir Turbulence in HF Heating of the Ionsphere," Phys. Rev. Lett., 62, 2676 (1989).

[2] F. T. Djuth, M. P. Sulzer and J. H. Elder, "High-Resolution Observations of the HFInduced Plasma Waves in the Ionosphere," Geophys. Res. Lett., 17, 1893 (1990).

[3] J. A. Fejer, M. P. Sulzer and F. T. Djuth, "Height Dependence of the Observed Spectrum of Radar Backscatter from HF-Induced Ionospheric Langmuir Turbulence," J. Geophys. Res., 15, 985 (1991).

[4] P. Y. Cheung, D. F. DuBois, T. Fukuchi, K. Kwan, Harvey A. Rose, David Russell, T. Tanikawa and A. Y. Wong, "Investigation of Strong Langmuir Turbulence in Ionospheric Modification," J. Geophys. Res., 97, 10575 (1992). 
[5] D. F. DuBois, H. A. Rose and D. Russell, "Power Spectra of Fluctuations in Strong Langmuir Turbulence," Phys. Rev. Lett., 61, 2209 (1988).

[6] D. F. DuBois, H. A. Rose and D. Russell, "Coexistence of Parametric Decay Cascades and Caviton Collapse at Subcritical Densities," Phys. Rev. Lett., 66, 1970 (1991).

[7] D. F. DuBois, H. A. Rose and D. Russell, "Excitation of Strong Langmuir Turbulence Near Critical Density: Application to HF Heating of the Ionsphere," J. Geophys. Res., 95, 21221 (1990).

[8] G. D. Doolen, D. F. DuBois and H. A. Rose, "Nucleation of Cavitons in Strong Langmuir Turbulence," Phys. Rev. Lett., 54, 804 (1985).

[9] D. Russell, D. F. DuBois and H. A. Rose, "Nucleation in Two-Dimensional Langmuir Turbulence," Phys. Rev. Lett., 60, 581 (1988).

[10] D. Russell, D. F. DuBois and H. A. Rose, "Collapsing Caviton Turbulence in One Dimension," Phys. Rev. Lett., 56, 838 (1986).

[11] D. F. DuBois, H. A. Rose and D. Russell, "Concepts in Strong Langmuir Turbulence," Proceedings of Summer Institute in Theoretical Physics on Nonlinear and Chaotic Phenomena in Plasmas, Solids and Fluids, U. of Alberta, Canada. Published by World Scientific, pp. 266-290 (1991).

[12] V. E. Zakharov, "Collapse of Langmuir Waves," Sov. Phys. JETP Eng. Transl., 35, 908 (1972).

[13] A. I. Dyachenko, A. M. Rubenchik, R. Z. Sagdeev, V. F. Shoets and V. E. Zakharov, "Computer Simulation of the Langmuir Collapse of the Isolated Cavity," in Plasma Theory and Nonlinear and Turbulent Processes in Physics, Vol. 2, edited by V. G. Baryakhtar et al., pp. 573-596 World Scientific, Hong Kong (1976).

[14] A. Hanssen, D. F. DuBois, E. Mjo lhus and Harvey A. Rose, "Numerical Test of the Weak Turbulence Approximation to Ionospheric Langmuir Turbulence," J. Geophys. Res., 97, 12073 (1992).

[15] D. F. DuBois, H. A. Rose and D. Russell, "Caviton Dynamics in Strong Langmuir Turbulence," Physica Scripta, T30, 137 (1990).

[16] D. F. DuBois, A. Hanssen, H. A. Rose and R. Russell, "Space and Time Distribution of HF Excited Langmuir Turbulence in the Ionosphere: Comparison of Theory and Experiment," J. Geophys. Res., 98, 17543 (1993). 